EPJ Web of Conferences 59, 10002 (2013)

DOI: $10.1051 /$ epjconf/20135910002

(C) Owned by the authors, published by EDP Sciences, 2013

\title{
Developments of compact pulsed-power system toward X-ray sources
}

\author{
Takuya Miyamoto, Fumitaka Tachinami, Toru Sasaki, Takashi Kikuchi \\ and Nob. Harada
}

Nagaoka University of Technology, Kamitomioka 1603-1, Nagaoka, Niigata 940-2188, Japan

\begin{abstract}
In order to generate X-rays from X-pinch, the peak current and current-rising time required are estimated to be $100 \mathrm{kA}$ and $100 \mathrm{~ns}$, respectively. To obtain these parameters, we developed a pulsed-power system, which consists of a parallelized pulse-forming network (PFN). The 20 PFN modules of the system were driven at a charging voltage of $20 \mathrm{kV}$ by a thin copper wire of load resistance. The results showed that the current and current-rising time are $18 \mathrm{kA}$ and $107 \mathrm{~ns}$, respectively. The wire/plasma temperature is $6.9 \mathrm{eV}$. The pulsed-power system is expected to generate $\mathrm{X}$-rays from $\mathrm{X}$-pinch by the proposed system. This can be achieved by raising the voltage and increasing the number of PFN modules.
\end{abstract}

\section{INTRODUCTION}

Warm dense matter (WDM) and other phenomena of high-energy-density physics (HEDPs) are generated by small experimental devices, which include compact-pulsed-power systems, ultra-shortpulse lasers, and intense ion beams. The generated HED matter is optically thick for the visible light, and this state of matter also has short lifetime. For example, WDM has high density $\left(0.01 \rho_{\mathrm{s}}-\rho_{\mathrm{s}}\right)$ and low temperature $\left(10^{3}-10^{5} \mathrm{~K}\right)$. By understanding the interior of HED matter in laboratory experiments, we can create a compact X-ray source with intense emissions. This intense X-ray source can be used to produce an X-pinch, which implodes wire/plasma driven by a pulsed-power system.

An X-pinch is formed by two wires that cross at a single point and is driven by a large current. A magnetic field is generated around the axis of wires when current flows through them. Magnetic pressure compresses the wire/plasma, and it can be further imploded by self-magnetic pressure. Radiation energy $W_{r}>500 \mathrm{~mJ}$ was observed in the range 1.2-1.5 keV and $W_{r}>20 \mathrm{~mJ}$ in the energy range 3-5 keV [1,2].

To generate X-rays from X-pinch, the required peak current and current-rising time are estimated to be $100 \mathrm{kA}$ and $100 \mathrm{~ns}$, respectively [3]. To obtain these parameters, we developed a pulsed-power module that consists of parallelized pulse-forming network (PFN).

\section{DESIGN OF PULSED-POWER SYSTEM}

To create an X-ray source using X-pinch, a pulse-power system requires a current of $100 \mathrm{kA}$ with a rise time of $100 \mathrm{~ns}$. The pulsed-power system consists of parallelized PFN modules, as shown in Fig. 1. A PFN consists of a series of high-voltage-energy-storage capacitors and inductors. These components are interconnected as a ladder network that behaves similarly to a length of transmission line. The circuit of a PFN module is shown in Fig. 2. The PFN module consists of two single PFNs in a parallel construction using $2000 \mathrm{pF}$ capacitors. The number of LC ladders is decided by calculation using Alternative Transients Program/ElectroMagnetic Transients Program (ATP/EMTP). Figure 3 shows the current waveforms changing the number of LC ladders and PFN modules connected in parallel with

This is an Open Access article distributed under the terms of the Creative Commons Attribution License 2.0, which permits unrestricted use, distribution, and reproduction in any medium, provided the original work is properly cited. 


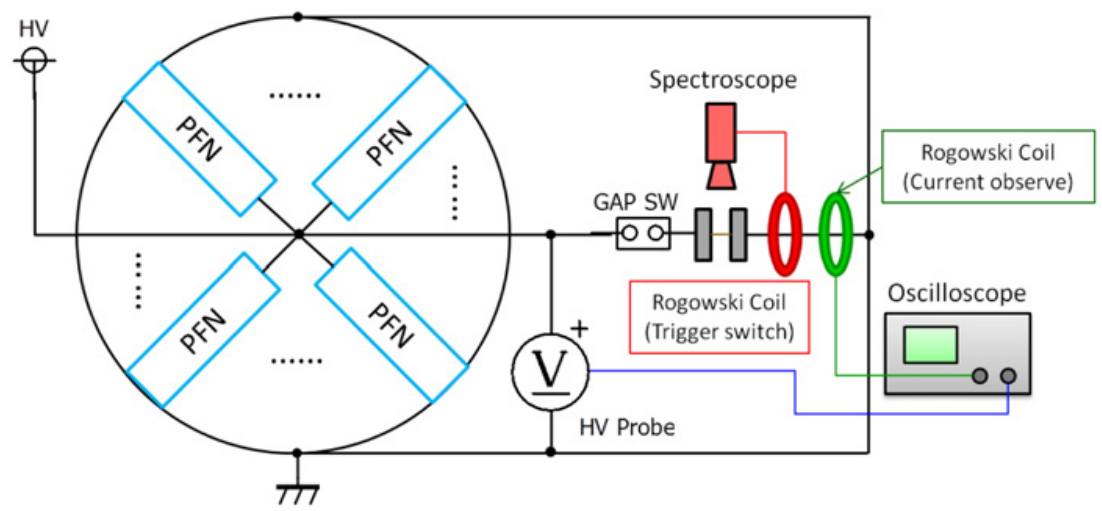

Figure 1. Schematic diagram of the experimental system.
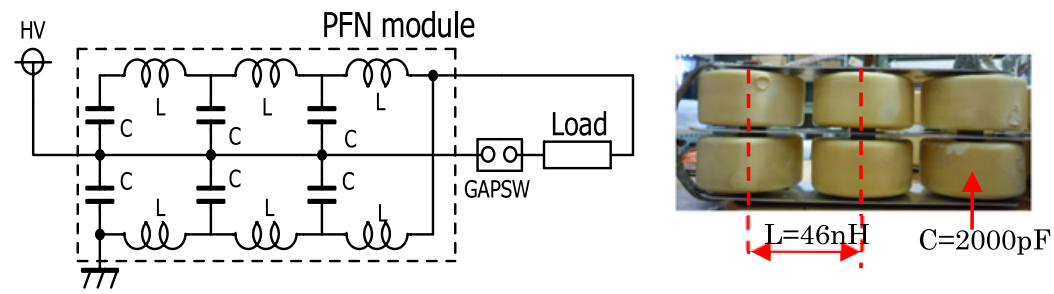

Figure 2. Circuit of PFN staged 3 LC ladders.

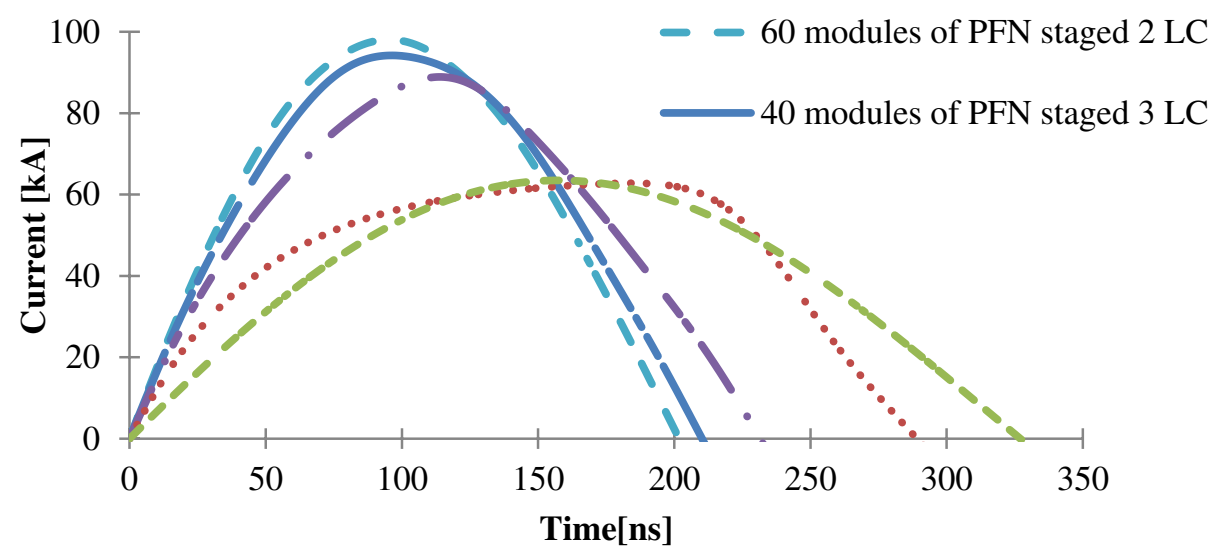

Figure 3. Current waveforms changing the number of LC ladders and PFN modules.

120 capacitors. The current-rising rate increases with an increase in the number of modules connected in parallel. The current-rising rate of $60 \mathrm{PFN}$ modules of staged $2 \mathrm{LC}$ ladders is $1.61 \times 10^{12} \mathrm{~A} / \mathrm{s}$. The current-rising rate of $40 \mathrm{PFN}$ modules of staged $3 \mathrm{LC}$ ladders is $1.52 \times 10^{12} \mathrm{~A} / \mathrm{s}$, which is $7 \%$ reduction in comparison with the case of 60 modules with staged 2 LC ladders. As the number of parallel modules increases, the size of the pulsed-power system increases. To create a compact system, we decided to utilize a PFN module consisting of three cascade LC ladders. The capacitors are connected to an aluminum plate. The middle of the plate is connected to a high-voltage power supply. The PFN 


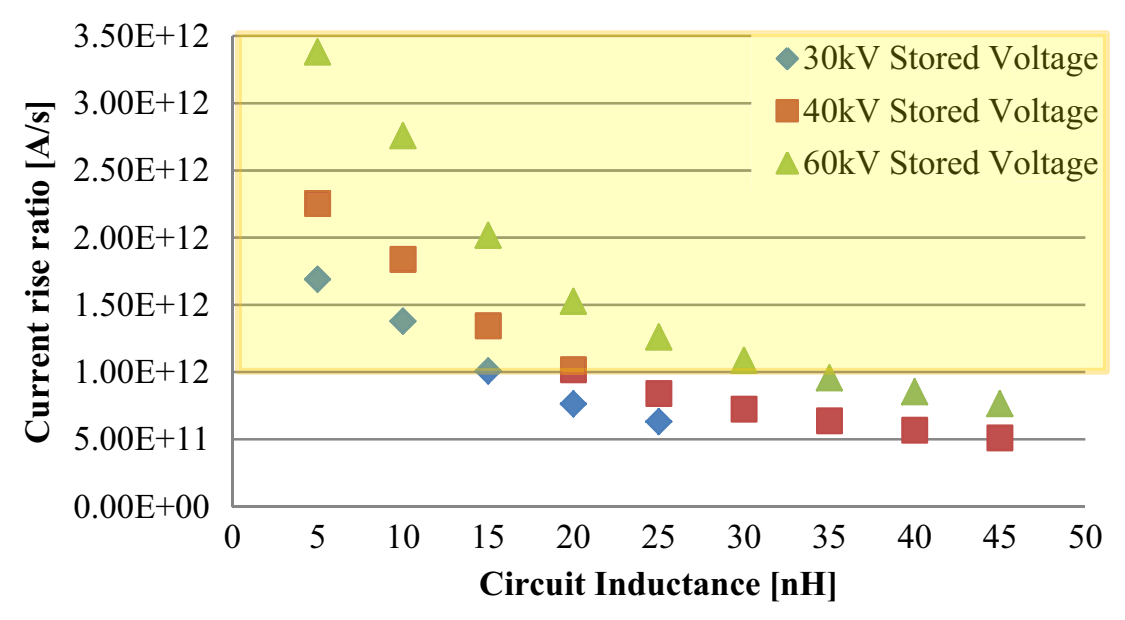

Figure 4. Allowed circuit inductance.

inductance between the capacitors is $52.3 \mathrm{nH}$. The PFN impedance is $5.11 \Omega$. The size of the PFN module is $60 \mathrm{~mm} \times 60 \mathrm{~mm} \times 200 \mathrm{~mm}$.

PFN modules connected in parallel can reduce the inductance of PFN and can increase the current. Therefore, the current-rising rate can be increased [4]. However, when there are more than 40 parallel PFN modules, the circuit inductance includes the load effect in the current-rising rate. To achieve current of $100 \mathrm{kA}$ with rising time of $100 \mathrm{~ns}$, the required circuit inductance is estimated by using ATP/EMTP. In the case of 40 parallel PFN modules of staged 3 LC ladders, the required circuit inductance is shown in Fig. 4. With a charging voltage of $30 \mathrm{kV}$, the circuit inductance should be less than $15 \mathrm{nH}$. The allowed inductance increases with an increase in charging voltage.

\section{DEMONSTRATIONS}

The experimental system is shown in Fig. 1. It consists of 20 PFN modules arranged in a circle, and $120 \mathrm{nF}$ capacitors at a charging voltage of $20 \mathrm{kV}$. The load is a fine copper wire with a length and diameter of $2 \mathrm{~mm}$ and $20 \mu \mathrm{m}$, respectively. The self-breakdown spark gap switch that is positioned in the middle of the modules, which are connected in a circle, is designed to operate in atmospheric air. A high voltage source is connected to the middle of the circle. The load current is measured at the cathode electrode using a Rogowski coil. The discharge voltage is measured at high-voltage plate of modules by using a high-voltage probe. The emission spectrum of ablation wire/plasma is measured at wire/plasma temperature by using a spectroscope mounted on a streak camera. The spectroscope is operated when a load current is detected using the Rogowski coil.

The voltage and current waveforms at load are shown in Fig. 5. The figure shows the peak voltage, current, and rise time to be $1.5 \mathrm{kV}, 18 \mathrm{kA}$, and $107 \mathrm{~ns}$, respectively. The current-rising rate is estimated to be $1.8 \times 10^{11} \mathrm{~A} / \mathrm{s}$. This result does not achieve the required parameters. However, the current-rising rate can be increased by increasing the charging voltage. The inductance of this system is estimated to be about $100 \mathrm{nH}$ by using an ATP/EMTP circuit simulation. To achieve current of $100 \mathrm{kA}$ with rising time of $100 \mathrm{~ns}$, the circuit inductance should be reduced.

Figure 6 shows the emission spectrum of copper wire/plasma at $202 \mathrm{~ns}$ from the beginning of discharge. The emission spectrum is assumed to be blackbody radiation. The wire/plasma temperature is estimated as $6.9 \mathrm{eV}$ in comparison with the Planck distribution. 


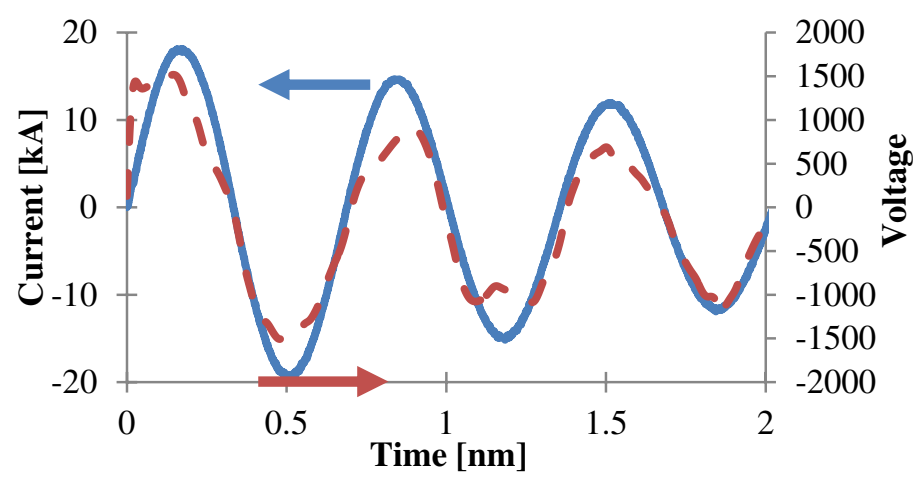

Figure 5. Typical voltage and current waveforms at load.

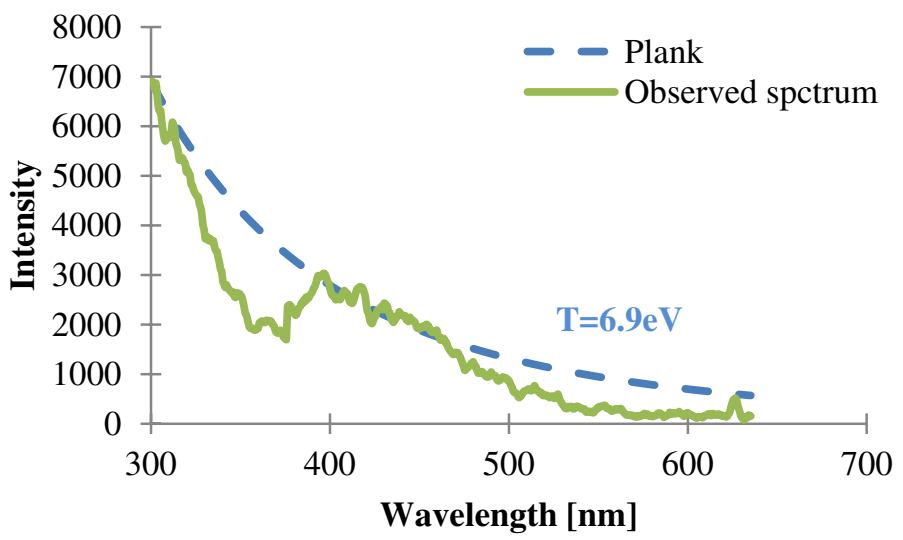

Figure 6. Emission spectrum of copper wire/plasma at $202 \mathrm{~ns}$ from the beginning of discharge. This emission spectrum can be assumed to be blackbody radiation. The wire/plasma temperature is estimated to be $6.9 \mathrm{eV}$ by the Planck formula.

\section{CONCLUSION}

To obtain a current of $100 \mathrm{kA}$ and rise time of $100 \mathrm{~ns}$, we developed a pulsed-power system that consists of PFN modules connected in parallel. The results of this experiment showed the peak current, rise time, and current-rising time to be $18 \mathrm{kA}, 112 \mathrm{~ns}$, and $1.8 \times 10^{11} \mathrm{~A} / \mathrm{s}$, respectively. The wire/plasma temperature was $6.9 \mathrm{eV}$. The pulsed-power system is expected to generate X-rays from X-pinch by the proposed system. This can be achieved by raising the voltage and increasing the number of PFN modules.

\section{References}

[1] A.V. Kharlov, et al., Rev. Sci. Instrum. 77, 123501 (2006)

[2] T.A. Shelkovenko, et al., Phys. Plasmas 12, 033102 (2005)

[3] L.S. Caballero and P.W. Smith, IEEE Trans. Plasma Sci. 37, No. 10, 1948 (2009)

[4] F. Tachinami, et al., IEEJ proceeding of national convention, P-8 (2011)

[5] L.E. Aranchuk, J. Larour, A.S. Chuvatin, IEEE Trans. Plasma Sci. 33, No. 2, 990 (2005) 